\title{
OVERVIEW OF THE DIFFERENTIAL GALOIS INTEGRABILITY CONDITIONS FOR NON-HOMOGENEOUS POTENTIALS
}

\author{
ANDRZEJ J. MACIEJEWSKI and MARIA PRZYBYLSKA \\ Institute of Astronomy and Institute of Physics, University of Zielona Góra, \\ Licealna 9, 65-417, Zielona Góra, Poland \\ E-mail: maciejka@astro.ia.uz.zgora.pl,M.Przybylska@proton.if.uz.zgora.pl
}

To Michael F. Singer on the occasion of his 60th birthday

\begin{abstract}
We report our recent results concerning integrability of Hamiltonian systems governed by Hamilton's function of the form $H=\frac{1}{2} \sum_{i=1}^{n} p_{i}^{2}+V(\boldsymbol{q})$, where the potential $V$ is a finite sum of homogeneous components. In this paper we show how to find, in the differential Galois framework, computable necessary conditions for the integrability of such systems. Our main result concerns potentials of the form $V=V_{k}+V_{K}$, where $V_{k}$ and $V_{K}$ are homogeneous functions of integer degrees $k$ and $K>k$, respectively. We present examples of integrable systems which were obtained by applying our main theorem.
\end{abstract}

1. Introduction. In our previous papers [7, 8, 14, 15] we investigated integrability of polynomial canonical systems given by Hamiltonian functions which have the form

$$
H=\frac{1}{2} \sum_{i=1}^{n} p_{i}^{2}+V_{k}(\boldsymbol{q}),
$$

where

$$
\boldsymbol{q}=\left(q_{1}, \ldots, q_{n}\right) \in \mathbb{C}^{n}, \quad \boldsymbol{p}=\left(p_{1}, \ldots, p_{n}\right) \in \mathbb{C}^{n},
$$

are the canonical coordinates and momenta, respectively, and $V_{k}$ is a homogeneous polynomial of degree $k \in \mathbb{Z}$. Our aim was to find all such systems which are integrable in the Liouville sense with meromorphic first integrals, and to show that the others are not integrable. We developed tools and methods which allowed us, to a large extent, to realise successfully this ambitious program for small $k>2$. Also we obtained some results concerning rational potentials.

2010 Mathematics Subject Classification: 37J30, 70Hxx.

Key words and phrases: differential Galois integrability obstructions, differential Galois theory, integrability, non-integrability, Hamilton systems.

The paper is in final form and no version of it will be published elsewhere. 
The next natural step is to consider the class of Hamilton equations with nonhomogeneous potentials. More precisely, in this paper we consider complex Hamiltonian systems with $n$ degrees of freedom governed by Hamilton function of the form

$$
H=\frac{1}{2} \sum_{i=1}^{n} p_{i}^{2}+V(\boldsymbol{q})
$$

We assume that the potential $V(\boldsymbol{q})$ is a finite sum of homogeneous components, that is,

$$
V(\boldsymbol{q}):=\sum_{i=k}^{K} V_{i}(\boldsymbol{q})
$$

where $V_{i}(\boldsymbol{q})$ is a homogeneous function of degree $i$. We say that $V$ is integrable iff the canonical equations

$$
\frac{\mathrm{d}}{\mathrm{d} t} \boldsymbol{q}=\boldsymbol{p}, \quad \frac{\mathrm{d}}{\mathrm{d} t} \boldsymbol{p}=-V^{\prime}(\boldsymbol{q}),
$$

where $V^{\prime}(\boldsymbol{q}):=\operatorname{grad} V(\boldsymbol{q})$, are integrable in the Liouville sense, i.e., they admit $n$ first integrals which are meromorphic and functionally independent in the prescribed domain and, moreover they pair-wise commute with respect to the canonical Poisson bracket. The integrability of such systems is important because many potentials interesting in physics are not homogeneous. Here we only mention two examples: the generalised Hénon-Heiles potential introduced in [2]

$$
V=\frac{1}{2}\left(A q_{1}^{2}+B q_{2}^{2}\right)+q_{1}^{2} q_{2}+\frac{\varepsilon}{3} q_{2}^{3},
$$

and the class of Yang-Mills-type potentials

$$
V=\frac{1}{2}\left(a_{1} q_{1}^{2}+a_{2} q_{2}^{2}\right)+\frac{1}{4} q_{1}^{4}+\frac{1}{4} a_{3} q_{2}^{4}+\frac{1}{2} a_{4} q_{1}^{2} q_{2}^{2},
$$

see [17] and references therein.

A classification of integrable homogeneous potentials was only possible thanks to very strong and effective conditions necessary for integrability. These conditions have been formulated quite recently and were obtained from the analysis of linear equations (socalled variational equations) which are the linearisation of canonical equations (4) along a non-equilibrium particular solution. The existence of first integrals of Hamilton equations (4) implies the existence of invariants of the monodromy as well as the differential Galois group of the variational equations. For basic notions and theorems of differential Galois theory see [16] and for the theory of monodromy group see e.g. [22]. For integrability in the Liouville sense, conditions obtained from the analysis of the differential Galois group of variational equations have an especially nice and useful form. In particular, the main theorem of the differential Galois approach to the integrability developed by Baider, Churchill, Morales, Ramis, Rod, Simó and Singer towards the end of the 20th century, see [12, 1], says, that if the system is integrable in the Liouville sense, then the identity component of the differential Galois group of variational equations is Abelian. For more details and references see, e.g., [12, 13].

For homogeneous potentials differential Galois integrability analysis has appeared very effective because for such potentials one can find, in a systematic way, particular 
solutions of equations (4). They have the form

$$
(\boldsymbol{q}(t), \boldsymbol{p}(t)):=(\varphi(t) \boldsymbol{d}, \dot{\varphi}(t) \boldsymbol{d}),
$$

where $\varphi(t)$ is a solution of $\ddot{\varphi}=-\varphi^{k-1}$, and a non-zero $\boldsymbol{d} \in \mathbb{C}^{n}$ is a solution of the system of non-linear equations

$$
V_{k}^{\prime}(\boldsymbol{d})=\alpha \boldsymbol{d}, \quad \alpha \in \mathbb{C} .
$$

Non-zero solutions of (5) are called Darboux points and in general this system has many solutions, so we have many particular solutions. Additionally, we can determine the differential Galois group of variational equations along such a particular solution. First of all, the variational equations split into a product of second order equations. Moreover, thanks to a clever Yoshida change of the independent variable [19], each of the second order equations can be transformed into the Gauss hypergeometric equation for which the differential Galois group is known. As a result, very useful necessary integrability conditions in the form of restrictions on eigenvalues of the Hessian $V_{k}^{\prime \prime}(\boldsymbol{d})$ were obtained, see 12 .

A possibility of more or less complete integrability analysis for non-homogeneous potentials depends on the existence of (many) non-trivial particular solutions. Moreover, it is equally important to have effective methods for determination of differential Galois groups of variational equations along these solutions.

It seems that the first step in such analysis was made by H. Ito in [3, 4]. He obtained necessary conditions for the integrability of polynomial potentials of degrees 3 and 4 from an analysis of the monodromy group of the variational equations. The techniques used in these papers do not have obvious generalisations to cases of polynomial potentials of arbitrary degrees or to non-polynomial potentials.

The next important result was obtained by H. Yoshida [20, 21] for non-homogeneous potentials with two degrees of freedom and later it was generalised to $n$ degrees of freedom by F. Mondejar [1]. It can be stated in the following form.

THEOREM 1.1 (Mondejar, Yoshida). Assume that Hamiltonian system (2) with potential of the form (3) satisfies the following conditions:

1. it admits a straight-line particular solution

$$
(\boldsymbol{q}(t), \boldsymbol{p}(t)):=(\varphi(t) \boldsymbol{d}, \dot{\varphi}(t) \boldsymbol{d})
$$

where $\boldsymbol{d} \in \mathbb{C}^{n} \backslash\{\mathbf{0}\}$, and $\varphi(t)$ is a non-constant scalar function,

2. it is integrable in the Liouville sense with first integrals meromorphic in a connected neighbourhood $U$ of the phase curve $\boldsymbol{\Gamma}$ corresponding to the above solution and functionally independent in $U \backslash \boldsymbol{\Gamma}$.

Then the truncated systems given by the Hamiltonian functions

$$
H_{k}=\frac{1}{2} \sum_{i=1}^{n} p_{i}^{2}+V_{k}(\boldsymbol{q}) \quad \text { and } \quad H_{K}=\frac{1}{2} \sum_{i=1}^{n} p_{i}^{2}+V_{K}(\boldsymbol{q})
$$

are integrable in the Liouville sense. Moreover, the eigenvalues $\left(\lambda_{1}^{(\kappa)}, \ldots, \lambda_{n}^{(\kappa)}\right)$ of the Hessian matrices $V_{\kappa}^{\prime \prime}(\boldsymbol{d})$ with $\kappa=k, K$ satisfy the following condition: each pair $\left(\kappa, \lambda_{j}^{(\kappa)}\right)$ with $\kappa=k, K$, and $j=1, \ldots, n$ belongs to one of the items from the following list: 


\begin{tabular}{cccc}
\hline case & $\kappa$ & $\lambda$ \\
\hline 1. & \pm 2 & arbitrary \\
2. & $\kappa$ & $p+\frac{\kappa}{2} p(p-1)$ & \\
3. & $\kappa$ & $\frac{1}{2}\left(\frac{\kappa-1}{\kappa}+p(p+1) \kappa\right)$ & \\
4. & 3 & $-\frac{1}{24}+\frac{1}{6}(1+3 p)^{2}$, & $-\frac{1}{24}+\frac{3}{32}(1+4 p)^{2}$ \\
& & $-\frac{1}{24}+\frac{3}{50}(1+5 p)^{2}$, & $-\frac{1}{24}+\frac{3}{50}(2+5 p)^{2}$ \\
5. & 4 & $-\frac{1}{8}+\frac{2}{9}(1+3 p)^{2}$ & \\
6. & 5 & $-\frac{9}{40}+\frac{5}{18}(1+3 p)^{2}$, & $-\frac{9}{40}+\frac{1}{10}(2+5 p)^{2}$ \\
7. & -3 & $\frac{25}{24}-\frac{1}{6}(1+3 p)^{2}$, & $\frac{25}{24}-\frac{3}{32}(1+4 p)^{2}$ \\
& & $\frac{25}{24}-\frac{3}{50}(1+5 p)^{2}$, & $\frac{25}{24}-\frac{3}{50}(2+5 p)^{2}$ \\
8. & -4 & $\frac{9}{8}-\frac{2}{9}(1+3 p)^{2}$ & \\
9. & -5 & $\frac{49}{40}-\frac{5}{18}(1+3 p)^{2}$, & $\frac{49}{40}-\frac{1}{10}(2+5 p)^{2}$ \\
\hline
\end{tabular}

where $p$ is an integer.

In order to prove this theorem an appropriate scaling of coordinates and time was made. This introduces a parameter into the problem. For the limiting cases of the parameter the problem reduces to integrability of the truncated systems with only the lowest, or highest, term in the potential expansion.

The question what can be added to the integrable Hamiltonian function without destructing its integrability has a long history and still is one of the most interesting problems of mechanics. KAM theorem says that most of the perturbations destroy integrability but does not give indications about possible integrable perturbations. As a rule, a modification of an integrable potential gives rise a non-integrable one. A typical modification is a truncation of non-homogeneous integrable potentials and this gives a non-integrable system. The first example of this effect is given in [20] where the non-integrability of truncated three-particle Toda lattice at any order was proved.

The weak point of Theorem 1.1 is that it does not put any restrictions on the terms $V_{i}$, with $k<i<K$. Moreover, although it seems that Theorem 1.1 is optimal for potentials which are sums of two homogeneous terms $V=V_{k}+V_{K}$, of degrees $k$ and $K$, respectively, it is not the case. Namely, a sum of two integrable potentials $V_{k}$ and $V_{K}$ can be non-integrable.

Our aim is to find necessary conditions for the integrability of non-homogeneous potentials which are stronger than those given by Theorem 1.1 . We show that in the case when $V$ is a sum of only two homogeneous terms one can find new necessary conditions for the integrability which are supplementary to those given by Theorem 1.1 . We formulate our result in the following form. 
MAIn THEOREM 1.2. Assume that a Hamiltonian system (2) satisfies the following conditions:

1. the potential $V$ is a sum of two homogeneous terms

$$
V=V_{k}+V_{K}
$$

where $k \in \mathbb{Z}^{\star}$ and $m=K-k \in \mathbb{N}$;

2. there exists a non-zero vector $\boldsymbol{d} \in \mathbb{C}^{n}$ such that

$$
V_{k}^{\prime}(\boldsymbol{d})=\alpha_{k} \boldsymbol{d}, \quad V_{K}^{\prime}(\boldsymbol{d})=\alpha_{K} \boldsymbol{d},
$$

for a certain non-zero $\alpha_{k}$ and $\alpha_{K}$.

3. both Hessian matrices $V_{k}^{\prime \prime}(\boldsymbol{d})$ and $V_{K}^{\prime \prime}(\boldsymbol{d})$ are simultaneously diagonalizable, and

$$
\boldsymbol{C}^{-1} V_{\kappa}^{\prime \prime}(\boldsymbol{d}) \boldsymbol{C}=\alpha_{\kappa} \operatorname{diag}\left(\lambda_{1}^{(\kappa)}, \ldots, \lambda_{n}^{(\kappa)}\right),
$$

for $\kappa=k, K$ and a certain non-singular matrix $\boldsymbol{C}$;

4. the system is integrable in the Liouville sense.

Then, for each $j \in\{1, \ldots, n\}$, either $\left(\lambda_{j}^{(k)}, \lambda_{j}^{(K)}\right)=\left(\lambda_{j}^{\left(\kappa_{1}\right)}, \lambda_{j}^{\left(\kappa_{2}\right)}\right)$, or $\left(\lambda_{j}^{(K)}, \lambda_{j}^{(k)}\right)=$ $\left(\lambda_{j}^{\left(\kappa_{1}\right)}, \lambda_{j}^{\left(\kappa_{2}\right)}\right)$, where $\lambda_{j}^{\left(\kappa_{1}\right)}$ and $\lambda_{j}^{\left(\kappa_{2}\right)}$ belong to an item of the following table:

\begin{tabular}{lll}
\hline case & $\lambda_{j}^{\left(\kappa_{1}\right)}$ & $\lambda_{j}^{\left(\kappa_{2}\right)}$ \\
\hline 1. & $\frac{1}{8 \kappa_{1}}\left[4 m^{2} r_{1}^{2}-\left(\kappa_{1}-2\right)^{2}\right]$ & $\frac{1}{8 \kappa_{2}}\left[4 m^{2} r_{2}^{2}-\left(\kappa_{2}-2\right)^{2}\right]$ \\
2. & $\frac{1}{8 \kappa_{1}}\left[m^{2}(2 l+1)^{2}-\left(\kappa_{1}-2\right)^{2}\right]$ & $\lambda$ \\
3. & $\frac{4 m^{2}(3 l+1)^{2}-9\left(\kappa_{1}-2\right)^{2}}{72 \kappa_{1}}$ & $\frac{4 m^{2}(3 p+1)^{2}-9\left(\kappa_{2}-2\right)^{2}}{72 \kappa_{2}}$ \\
4. & $\frac{4 m^{2}(3 l+1)^{2}-9\left(\kappa_{1}-2\right)^{2}}{72 \kappa_{1}}$ & $\frac{m^{2}(4 p+1)^{2}-4\left(\kappa_{2}-2\right)^{2}}{32 \kappa_{2}}$ \\
5. & $\frac{4 m^{2}(3 l+1)^{2}-9\left(\kappa_{1}-2\right)^{2}}{72 \kappa_{1}}$ & $\frac{4 m^{2}(5 p+1)^{2}-25\left(\kappa_{2}-2\right)^{2}}{200 \kappa_{2}}$ \\
6. & $\frac{4 m^{2}(5 l+2)^{2}-25\left(\kappa_{1}-2\right)^{2}}{200 \kappa_{1}}$ & $\frac{4 m^{2}(5 p+1)^{2}-25\left(\kappa_{2}-2\right)^{2}}{200 \kappa_{2}}$ \\
7. & $\frac{4 m^{2}(5 l+2)^{2}-25\left(\kappa_{1}-2\right)^{2}}{200 \kappa_{1}}$ & $\frac{4 m^{2}(3 p+1)^{2}-9\left(\kappa_{2}-2\right)^{2}}{72 \kappa_{2}}$ \\
\hline
\end{tabular}

where $\lambda \in \mathbb{C}, m=K-k, l, p$ are integers, and $r_{1}, r_{2}$ are rational numbers satisfying

$$
r_{1}+r_{2}=p+1 / 2 \quad \text { or } \quad r_{1}-r_{2}=p+1 / 2 \text {. }
$$

We deduce the above theorem from an analysis of the variational equations along a straight line solution whose existence is guaranteed by the second assumption. In fact, this solution is the same as in Theorem 1.1. But now we do not rescale coordinates and time in order to remove coupling of terms with various degrees of homogeneity.

The conditions given by our theorem put restrictions on pairs of eigenvalues $\lambda_{i}^{(k)}$ and $\lambda_{i}^{(K)}$ of the respective matrices $V_{k}^{\prime \prime}(\boldsymbol{d})$ and $V_{K}^{\prime \prime}(\boldsymbol{d})$, corresponding to the same eigenvector. Moreover, these conditions put simultaneous restrictions on both degrees of homogeneity $k$ and $K$. 
As we will explain in the next section, the problem of determination of the differential Galois group of the variational equations in the relevant problem is not, in general, tractable. However, under the third assumption of the theorem, the variational equations split into a product of second order equations. Additionally, on the zero energy level each of these second order equations can be transformed into a Riemann $P$ equation. We formulate a theorem that determines all the cases with Abelian identity of the differential Galois group for our variational equations. The proof of Theorem 1.2 consists in the application of this theorem.

Theorems 1.1 and 1.2 together give the strongest known necessary integrability conditions for non-homogeneous potentials consisting of two homogeneous parts. Comments about their applications together with the most interesting integrable examples with first integrals of degree four in the momenta are given in Section 4 All details are contained in the forthcoming article [9].

2. Discussion of the general case. Let us start from the general form of potential (3) and assume that there exists a non-zero solution $\boldsymbol{d} \in \mathbb{C}^{n}$ of the following system of non-linear equations

$$
V_{i}^{\prime}(\boldsymbol{d})=\alpha_{i} \boldsymbol{d}, \quad i=k, \ldots, K,
$$

with some nonzero $\alpha_{i} \in \mathbb{C}$.

The existence of $\boldsymbol{d}$ guarantees that Hamiltonian system (4) possesses a straight-line particular solution of the form

$$
\boldsymbol{q}(t)=\varphi(t) \boldsymbol{d}, \quad \boldsymbol{p}(t)=\dot{\varphi}(t) \boldsymbol{d},
$$

provided $\varphi$ is a solution of the scalar equation

$$
\ddot{\varphi}=-\sum_{i=k}^{K} \alpha_{i} \varphi^{i-1} .
$$

The variational equations along this particular solution are

$$
\left[\begin{array}{c}
\dot{\boldsymbol{Q}} \\
\dot{\boldsymbol{P}}
\end{array}\right]=\left[\begin{array}{cc}
\mathbf{0}_{n} & \boldsymbol{E}_{n} \\
-V^{\prime \prime}(\varphi \boldsymbol{d}) & \mathbf{0}_{n}
\end{array}\right]\left[\begin{array}{l}
\boldsymbol{Q} \\
\boldsymbol{P}
\end{array}\right],
$$

where $\boldsymbol{E}_{n}$ and $\mathbf{0}_{n}$ are the $n$-dimensional identity and zero matrix, respectively. As $V_{i}^{\prime \prime}(\boldsymbol{q})$ is a homogeneous function of degree $i-2$, we have

$$
V^{\prime \prime}(\varphi \boldsymbol{d})=\sum_{i=k}^{K} V_{i}^{\prime \prime}(\varphi \boldsymbol{d})=\sum_{i=k}^{K} \varphi^{i-2} V_{i}^{\prime \prime}(\boldsymbol{d}),
$$

and the variational equations 16 can be rewritten in the compact form

$$
\ddot{\boldsymbol{Q}}=-\left(\sum_{i=k}^{K} \varphi^{i-2}(t) V_{i}^{\prime \prime}(\boldsymbol{d})\right) \boldsymbol{Q} .
$$

We do not know how to determine in general the differential Galois group of this system of coupled equations. That is why we assume that all matrices $V_{i}^{\prime \prime}(\boldsymbol{d})$, for $i=k, \ldots, K$ are simultaneously diagonalisable, and

$$
\boldsymbol{C}^{-1} V_{i}^{\prime \prime}(\boldsymbol{d}) \boldsymbol{C}=\alpha_{i} \operatorname{diag}\left(\lambda_{1}^{(i)}, \ldots, \lambda_{n}^{(i)}\right) \quad \text { for } \quad i=k, \ldots, K,
$$


where $C$ is a certain non-singular matrix. This matrix determines the transformation $\boldsymbol{Q}=\boldsymbol{C} \boldsymbol{\eta}$ that splits variational equations into a direct product of the second order scalar equations

$$
\ddot{\eta}_{j}=-A_{j}(t) \eta_{j}, \quad \text { where } \quad A_{j}(t)=\sum_{i=k}^{K} \alpha_{i} \lambda_{j}^{(i)} \varphi^{i-2}(t), \quad j=1, \ldots, n .
$$

The second order autonomous equation (15) can be rewritten as a Hamiltonian system with one degree of freedom. Then the choice of the value of energy $e$ of this system determines a particular solution $\varphi(t)$. The corresponding phase curve is a hyperelliptic curve defined by

$$
\dot{\varphi}^{2}=2 e-2 \sum_{i=k}^{K} \frac{\alpha_{i}}{i} \varphi^{i}
$$

Each of equations 20 can be transformed into a second order linear equation with rational coefficients by the transformation

$$
t \rightarrow z:=\varphi(t) .
$$

Since

$$
\frac{\mathrm{d}^{2}}{\mathrm{~d} t^{2}}=\dot{z}^{2} \frac{\mathrm{d}^{2}}{\mathrm{~d} z^{2}}+\ddot{z} \frac{\mathrm{d}}{\mathrm{d} z},
$$

after transformation (22) variational equations take the form

$$
\eta_{j}^{\prime \prime}+p \eta_{j}^{\prime}+q_{j} \eta_{j}=0, \quad j=1, \ldots, n, \quad ' \equiv \frac{\mathrm{d}}{\mathrm{d} z},
$$

with coefficients

$$
p=\frac{\ddot{z}}{\dot{z}^{2}}=\frac{1}{2} \frac{P^{\prime}(z)}{P(z)}, \quad q_{j}=\frac{A_{j}(t)}{\dot{z}^{2}}=\frac{Q_{j}(z)}{P(z)},
$$

where

$$
P(z):=2 e-2 \sum_{i=k}^{K} \frac{\alpha_{i}}{i} z^{i}, \quad \text { and } \quad Q_{j}(z):=\sum_{i=k}^{K} \alpha_{i} \lambda_{j}^{(i)} z^{i-2} \quad \text { for } \quad j=1, \ldots n .
$$

If the system (4) is integrable in the Liouville sense, then the identity component of the differential Galois group of variational equations 20 as well as equations 23 is Abelian. The differential Galois group of the system 23 is contained in the product of the differential Galois groups of each of these equations. If for one of these second-order equations the identity component is not Abelian, then the identity component of the whole system (23) is not Abelian.

Determination of the differential Galois group of the second order linear equation with rational coefficient is in principle always possible. There exists an effective closed algorithm, the so-called Kovacic algorithm [6], which solves this problem. In fact, using it we can decide if the differential Galois group of the considered equation is virtually Abelian. However, in practice, it can be done effectively only for equations without parameters. But the nature of the formulated problem is such that we have a lot of parameters because we want to distinguish all integrable potentials. 
Here we want to point out that an equation of the form $(23)$ with coefficients given by (24) has several remarkable properties. For example, it is possible to prove that for generic values of the energy, the monodromy group of this equation is a reflection group.

3. Sketch of the proof of the main theorem. We recall that in our main theorem we assumed that the potential $V$ is a sum of only two terms

$$
V=V_{k}+V_{K}
$$

where $V_{k}$ and $V_{K}$ are homogeneous potentials of integer degrees $k \neq 0$ and $K \neq 0$, respectively, and $K-k=m \geq 1$. Moreover, we assumed also existence of a non-zero solution $\boldsymbol{d}$ of the systems

$$
V_{k}^{\prime}(\boldsymbol{d})=\alpha_{k} \boldsymbol{d}, \quad V_{K}^{\prime}(\boldsymbol{d})=\alpha_{K} \boldsymbol{d},
$$

with $\alpha_{k}, \alpha_{K} \in \mathbb{C}^{\star}:=\mathbb{C} \backslash\{0\}$. And finally we assumed existence of a non-singular matrix C such that

$$
\boldsymbol{C}^{-1} V_{\kappa}^{\prime \prime}(\boldsymbol{d}) \boldsymbol{C}=\alpha_{\kappa} \operatorname{diag}\left(\lambda_{1}^{(\kappa)}, \ldots, \lambda_{n}^{(\kappa)}\right),
$$

for $\kappa \in\{k, K\}$. Hence, in this case, the coefficients of variational equations 23] simplify to the form

$$
p=\frac{k K z^{k}\left(\alpha_{K} z^{m}+\alpha_{k}\right)}{2 z\left(k \alpha_{K} z^{K}+K \alpha_{k} z^{k}-e k K\right)}, \quad q_{j}=-\frac{k K z^{k}\left(\alpha_{K} \lambda_{j}^{(K)} z^{m}+\alpha_{k} \lambda_{j}^{(k)}\right)}{2 z^{2}\left(k \alpha_{K} z^{K}+K \alpha_{k} z^{k}-e k K\right)},
$$

where $m=K-k$, and $j \in\{1, \ldots, n\}$.

Let us note that the variational equations depend on a chosen value $e$. For a generic value of $e$, the number of singularities of these equations is big. For example, for $k \geq 2$, each of the variational equations has $K+1$ singularities. This is why analysis of these equations with a generic value of $e$ is highly complicated as was already mentioned above. However, for $e=0$ the coefficients $p$ and $q_{j}$ simplify considerably

$$
p=\frac{k K\left(\alpha_{K} z^{m}+\alpha_{k}\right)}{2 z\left(k \alpha_{K} z^{m}+K \alpha_{k}\right)}, \quad q_{j}=-\frac{k K\left(\alpha_{K} \lambda_{j}^{(K)} z^{m}+\alpha_{k} \lambda_{j}^{(k)}\right)}{2 z^{2}\left(k \alpha_{K} z^{m}+K \alpha_{k}\right)} .
$$

Hence, for $e=0$, each of the variational equations has $m+2$ singularities. Moreover, we simplify them further introducing new independent variable

$$
x=-\frac{k \alpha_{K}}{K \alpha_{k}} z^{m} .
$$

After this transformation the $j$-th variational equation in $(23)$ takes the form

$$
\frac{\mathrm{d}^{2} \eta}{\mathrm{d} x^{2}}+\left(\frac{k+2 m-2}{2 m x}+\frac{1}{2(x-1)}\right) \frac{\mathrm{d} \eta}{\mathrm{d} x}+\left(-\frac{k \lambda_{j}^{(k)}}{2 m^{2} x^{2}}+\frac{k \lambda_{j}^{(k)}-K \lambda_{j}^{(K)}}{2 m^{2} x(x-1)}\right) \eta=0 .
$$

So, it is a Riemann $P$ equation in the standard form, see e.g. [18]. In fact, if we rewrite it in the form

$$
\begin{aligned}
\frac{\mathrm{d}^{2} \eta}{\mathrm{d} x^{2}}+\left(\frac{1-\alpha_{1}-\alpha_{2}}{x}+\frac{1-\beta_{1}-\beta_{2}}{x-1}\right) & \frac{\mathrm{d} \eta}{\mathrm{d} x} \\
& \quad+\left(\frac{\alpha_{1} \alpha_{2}}{x^{2}}+\frac{\beta_{1} \beta_{2}}{(x-1)^{2}}+\frac{\gamma_{1} \gamma_{2}-\alpha_{1} \alpha_{2}-\beta_{1} \beta_{2}}{x(x-1)}\right) \eta=0
\end{aligned}
$$


we find out that

$$
\begin{aligned}
& \alpha_{1}=\frac{1}{4 m}\left(2-k+\sqrt{(k-2)^{2}+8 k \lambda_{j}^{(k)}}\right), \quad \alpha_{2}=\frac{1}{4 m}\left(2-k-\sqrt{(k-2)^{2}+8 k \lambda_{j}^{(k)}}\right), \\
& \gamma_{1}=\frac{1}{4 m}\left(K-2+\sqrt{(K-2)^{2}+8 K \lambda_{j}^{(K)}}\right), \quad \beta_{1}=\frac{1}{2}, \\
& \gamma_{2}=\frac{1}{4 m}\left(K-2-\sqrt{(K-2)^{2}+8 K \lambda_{j}^{(K)}}\right), \quad \beta_{2}=0 .
\end{aligned}
$$

The differences of the exponents at the singular points $x=0, x=1$ and $x=\infty$ are respectively

$$
\begin{aligned}
& \alpha=\alpha_{1}-\alpha_{2}=\frac{1}{2 m} \sqrt{(k-2)^{2}+8 k \lambda_{j}^{(k)}}, \quad \beta=\beta_{1}-\beta_{2}=\frac{1}{2}, \\
& \gamma=\gamma_{1}-\gamma_{2}=\frac{1}{2 m} \sqrt{(K-2)^{2}+8 K \lambda_{j}^{(K)}} .
\end{aligned}
$$

The reduced form of equation 32 is

$$
\frac{\mathrm{d}^{2} v}{\mathrm{~d} z^{2}}+\frac{1}{4}\left\{\frac{1-\alpha^{2}}{z^{2}}+\frac{1-\beta^{2}}{(z-1)^{2}}-\frac{1-\alpha^{2}-\beta^{2}+\gamma^{2}}{z(z-1)}\right\} v=0 .
$$

We denote by $\left(\rho_{1}, \rho_{2}\right),\left(\sigma_{1}, \sigma_{2}\right)$ and $\left(\tau_{1}, \tau_{2}\right)$ the exponents at the respective singular points $z=0, z=1$ and $z=\infty$, for this equation. One can find that

$$
\rho_{1,2}=\frac{1}{2}(1 \pm \alpha), \quad \sigma_{1,2}=\frac{1}{2}(1 \pm \beta), \quad \tau_{1,2}=-\frac{1}{2}(1 \mp \gamma) .
$$

Let us notice that the differences of exponents for the reduced Riemann equation are the same as for equation 32 , i.e.,

$$
\rho:=\rho_{1}-\rho_{2}=\alpha, \quad \sigma:=\sigma_{1}-\sigma_{2}=\beta, \quad \tau:=\tau_{1}-\tau_{2}=\gamma .
$$

Let us assume that the potential is integrable. Hence, by differential Galois integrability obstructions, the identity component of the differential Galois group of the variational equations (31) with $j=1, \ldots, n$, is Abelian. Thus, our task is to determine necessary and sufficient conditions imposed on the parameters $k, K$ and $\lambda_{j}$ which guarantee that this is the case. For the Riemann $P$ equation the Kimura theorem, see [5], specifies all the values of the parameters of this equation for which the identity component of the differential Galois group is solvable. However, a solvable group is not necessarily Abelian. This is why, first, we have to find all the parameters' values for which the differential Galois group of the Riemann $P$ equation is actually Abelian. They are specified in the following theorem.

THEOREM 3.1. The identity component of the reduced Riemann $P$ equation (34) is Abelian if and only if either

A: at least one of four numbers $\rho+\sigma+\tau,-\rho+\sigma+\tau, \rho-\sigma+\tau, \rho+\sigma-\tau$, is an odd integer, and moreover either two among numbers $\rho, \sigma$ are rational, or at least one among numbers $\rho, \sigma, \tau$ is an integer and there is no logarithmic singularity; or

$B$ : the numbers $\rho$ or $-\rho$ and $\sigma$ or $-\sigma$ and $\tau$ or $-\tau$ belong (in an arbitrary order) to some of the following fifteen families: 


\begin{tabular}{|c|c|c|c|l|}
\hline 1 & $1 / 2+l$ & $1 / 2+s$ & arbitrary complex number & \\
\hline 2 & $1 / 2+l$ & $1 / 3+s$ & $1 / 3+q$ & \\
\hline 3 & $2 / 3+l$ & $1 / 3+s$ & $1 / 3+q$ & $l+s+q$ even \\
\hline 4 & $1 / 2+l$ & $1 / 3+s$ & $1 / 4+q$ & \\
\hline 5 & $2 / 3+l$ & $1 / 4+s$ & $1 / 4+q$ & $l+s+q$ even \\
\hline 6 & $1 / 2+l$ & $1 / 3+s$ & $1 / 5+q$ & \\
\hline 7 & $2 / 5+l$ & $1 / 3+s$ & $1 / 3+q$ & $l+s+q$ even \\
\hline 8 & $2 / 3+l$ & $1 / 5+s$ & $1 / 5+q$ & $l+s+q$ even \\
\hline 9 & $1 / 2+l$ & $2 / 5+s$ & $1 / 5+q$ & \\
\hline 10 & $3 / 5+l$ & $1 / 3+s$ & $1 / 5+q$ & $l+s+q$ even \\
\hline 11 & $2 / 5+l$ & $2 / 5+s$ & $2 / 5+q$ & $l+s+q$ even \\
\hline 12 & $2 / 3+l$ & $1 / 3+s$ & $1 / 5+q$ & $l+s+q$ even \\
\hline 13 & $4 / 5+l$ & $1 / 5+s$ & $1 / 5+q$ & $l+s+q$ even \\
\hline 14 & $1 / 2+l$ & $2 / 5+s$ & $1 / 3+q$ & $l+s+q$ even \\
\hline 15 & $3 / 5+l$ & $2 / 5+s$ & $1 / 3+q$ & \\
\hline
\end{tabular}

where $l, s, q \in \mathbb{Z}$.

For the proof of this auxiliary theorem, see [9]. Now, to prove our Theorem 1.2 it is enough to apply, case by case, Theorem 3.1 to equation (34). For details, see [9].

4. Applications and remarks. Let us consider a potential of the form

$$
V=A q_{1}^{2}+B q_{2}^{2}+V_{\kappa}\left(q_{1}, q_{2}\right), \quad A, B \in \mathbb{C},
$$

where $V_{\kappa}$ is a homogeneous potential of integer degree $\kappa \neq 2$. For $\kappa=3$ and $\kappa=4$ we know all integrable polynomial potentials, see [7, 8]. This is why we can apply our Theorem 1.2. Here we give four examples of integrable potentials which were found in this way and which admit an additional first integral of degree 4 with respect to the momenta. Two of them are examples of harmonic perturbations of cubic integrable potentials

$$
\begin{aligned}
& V=\frac{1}{2} q_{1}^{2} q_{2}+\frac{8}{3} q_{2}^{3}+\frac{a}{2}\left(q_{1}^{2}+16 q_{2}^{2}\right), \\
& I=18 p_{1}^{4}-12 p_{1} p_{2} q_{1}^{3}+36 p_{1}^{2} q_{1}^{2} q_{2}-q_{1}^{4}\left(q_{1}^{2}+6 q_{2}^{2}\right)+6 a q_{1}^{2}\left(6 p_{1}^{2}+q_{1}^{2}\left(3 a-2 q_{2}\right)\right),
\end{aligned}
$$

and

$$
\begin{aligned}
& V=\frac{\mathrm{i} \sqrt{3}}{18} q_{1}^{3}+\frac{1}{2} q_{1}^{2} q_{2}+q_{2}^{3}+\frac{a}{2}\left(q_{1}^{2}+6 q_{2}^{2}\right), \\
& I=36 \sqrt{3} p_{1}^{4}+72 \mathrm{i} p_{1}^{3} p_{2}-36 p_{1} p_{2} q_{1}\left(-6 \sqrt{3} a^{2}+3 \mathrm{i} a q_{1}+q_{1}\left(\sqrt{3} q_{1}+3 \mathrm{i} q_{2}\right)\right)+1296 \sqrt{3} a^{4} q_{2}^{2} \\
& +18 \mathrm{i} a q_{1}^{3}\left(q_{1}^{2}+\mathrm{i} \sqrt{3} q_{1} q_{2}+6 q_{2}^{2}\right)+9 \sqrt{3} a^{2} q_{1}^{2}\left(7 q_{1}^{2}+12 q_{2}^{2}\right)+36 p_{1}^{2}\left(\mathrm{i} q_{1}^{3}-6 \sqrt{3} a^{2} q_{2}\right. \\
& \left.+2 \sqrt{3} q_{1}^{2}\left(a+q_{2}\right)+6 \mathrm{i} q_{1} q_{2}\left(2 a+q_{2}\right)\right)+216 \sqrt{3} a^{3}\left(p_{2}^{2}+2 q_{2}\left(q_{1}^{2}+q_{2}^{2}\right)\right)+q_{1}^{3}\left(-5 \sqrt{3} q_{1}^{3}\right. \\
& \left.+18 \mathrm{i} q_{1}^{2} q_{2}-9 \sqrt{3} q_{1} q_{2}^{2}+36 \mathrm{i}\left(p_{2}^{2}+q_{2}^{3}\right)\right) .
\end{aligned}
$$

The other two are examples of harmonic perturbations of quartic integrable potentials

$$
\begin{aligned}
& V=\frac{4}{3} q_{1}^{4}+q_{1}^{2} q_{2}^{2}+\frac{1}{6} q_{2}^{4}+\frac{a}{2}\left(4 q_{1}^{2}+q_{2}^{2}\right), \\
& I=9 p_{2}^{4}+12 p_{2} q_{1} q_{2}^{2}\left(3 p_{2} q_{1}-2 p_{1} q_{2}\right)+6\left(p_{1}^{2}+p_{2}^{2}\right) q_{2}^{4}+18 b p_{2}^{2} q_{2}^{2}+q_{2}^{4}\left(3 b+2 q_{1}^{2}+q_{2}^{2}\right)^{2},
\end{aligned}
$$


and

$$
\begin{aligned}
& V=\frac{1}{32} q_{1}^{4}+\frac{3}{16} q_{1}^{2} q_{2}^{2}+\frac{1}{4} q_{2}^{4}+\frac{a}{2}\left(q_{1}^{2}+4 q_{2}^{2}\right), \\
& I=256 p_{1}^{4}+32 p_{2}^{2} q_{1}^{4}+q_{1}^{8}-128 p_{1} p_{2} q_{1}^{3} q_{2}+32 p_{1}^{2}\left(q_{1}^{4}+6 q_{1}^{2} q_{2}^{2}\right)+4 q_{1}^{4} q_{2}^{2}\left(q_{1}^{2}+q_{2}^{2}\right) \\
& +32 a q_{1}^{2}\left(16 p_{1}^{2}+q_{1}^{2}\left(8 a+q_{1}^{2}+2 q_{2}^{2}\right)\right) .
\end{aligned}
$$

Let us consider a generalisation of potential (37) to $n$ degrees of freedom

$$
V=\frac{1}{2} \boldsymbol{q}^{T} \boldsymbol{S} \boldsymbol{q}+V_{\kappa}(\boldsymbol{q}), \quad \boldsymbol{S}=\boldsymbol{S}^{T}
$$

Then, we meet the following two problems with application of Theorem 1.2. It is easy to check that the second assumption is equivalent to the following condition: the potential $V_{\kappa}$ has a Darboux point which is an eigenvector of matrix $\boldsymbol{S}$. Moreover, even if we assume that this is the case we have another problem. The matrices $\boldsymbol{S}$ and $V_{\kappa}^{\prime \prime}(\boldsymbol{d})$ for $n>2$ are not, in general, simultaneously diagonalisable. Hence, the variational equations do not split into a direct product of second order variational equations. For $n=2$ if $V_{k}$ and $V_{K}$ have a common Darboux point $\boldsymbol{d}=\left(d_{1}, d_{2}\right)$ such that $d_{1}^{2}+d_{2}^{2} \neq 0$, then the Hessian matrices $V_{k}^{\prime \prime}(\boldsymbol{d})$ and $V_{K}^{\prime \prime}(\boldsymbol{d})$ are simultaneously diagonalisable. More about this problem can be found in 9 .

Clearly, we do not have problems with a common Darboux point in the case of homogeneous perturbations of $n$-dimensional radial potentials, i.e., when the potential has the form

$$
V=\alpha r^{2 s}+V_{\kappa}(\boldsymbol{q}), \quad r^{2}=\sum_{i=1}^{n} q_{i}^{2},
$$

where $2 s \in \mathbb{Z}$, and $V_{\kappa}$ is a homogeneous potential of integer degree $\kappa \neq 2 s$. In this case, if we want to apply Theorem 1.2 it is enough to assume that $V_{\kappa}$ has a Darboux point $\boldsymbol{d}$, and $V_{\kappa}^{\prime \prime}(\boldsymbol{d})$ is diagonalisable. An integrability analysis of such potentials will be published in [10].

Acknowledgments. The authors are grateful to European Science Foundation (ESF) for grants supporting their participation in the conference "Algebraic Methods in Dynamical Systems" in Będlewo.

\section{References}

[1] A. Baider, R. C. Churchill, D. L. Rod and M. F. Singer, On the infinitesimal geometry of integrable systems, in: Mechanics Day (Waterloo, ON, 1992), Fields Inst. Commun. 7, Amer. Math. Soc., Providence, RI, 1996, 5-56.

[2] M. Hénon and C. Heiles, The applicability of the third integral of motion: Some numerical experiments, Astronom. J. 69 (1964), 73-79.

[3] H. Ito, Non-integrability of Hénon-Heiles system and a theorem of Ziglin, Kodai Math. J. 8 (1985), 120-138.

[4] H. Ito, A criterion for non-integrability of Hamiltonian systems with non-homogeneous potentials, Z. Angew. Math. Phys. 38 (1987), 459-476.

[5] T. Kimura, On Riemann's equations which are solvable by quadratures, Funkcial. Ekvac. $12(1969 / 1970), 269-281$. 
[6] J. J. Kovacic, An algorithm for solving second order linear homogeneous differential equations, J. Symbolic Comput. 2 (1986), 3-43.

[7] A. J. Maciejewski and M. Przybylska, All meromorphically integrable 2D Hamiltonian systems with homogeneous potentials of degree 3, Phys. Lett. A 327 (2004), 461-473.

[8] A. J. Maciejewski and M. Przybylska, Darboux points and integrability of Hamiltonian systems with homogeneous polynomial potential, J. Math. Phys. 46 (2005), 062901, 33 pages.

[9] A. J. Maciejewski and M. Przybylska, Integrable deformations of integrable Hamiltonian systems, Phys. Lett. A 376 (2011), 80-93.

[10] A. J. Maciejewski and M. Przybylska, Integrable perturbations of radial potentials, Celestial Mech. Dynam. Astronom., in preparation.

[11] F. Mondéjar, S. Ferrer and A. Vigueras, On the non-integrability of Hamiltonian systems with sum of homogeneous potentials, Technical report, Departamento de Matematica Aplicada y Estadistica, Universidad Politecnica de Cartagena, 1999.

[12] J. J. Morales Ruiz, Differential Galois Theory and Non-Integrability of Hamiltonian Systems, Progr. Math. 179, Birkhäuser, Basel, 1999.

[13] J. J. Morales Ruiz and J.-P. Ramis, Integrability of dynamical systems through differential Galois theory: a practical guide, in: Differential Algebra, Complex Analysis and Orthogonal Polynomials, Contemp. Math. 509, Amer. Math. Soc., Providence, RI, 143-220.

[14] M. Przybylska, Darboux points and integrability of homogenous Hamiltonian systems with three and more degrees of freedom, Regul. Chaotic Dyn. 14 (2009), 263-311.

[15] M. Przybylska, Darboux points and integrability of homogenous Hamiltonian systems with three and more degrees of freedom. Nongeneric cases, Regul. Chaotic Dyn. 14 (2009), 349-388.

[16] M. van der Put and M. F. Singer, Galois Theory of Linear Differential Equations, Grundlehren der Mathematischen Wissenschaften 328, Springer-Verlag, Berlin, 2003.

[17] M. E. Sansaturio, I. Vigo-Aguiar and J. M. Ferrándiz, Non-integrability of some Hamiltonian systems in polar coordinates, J. Phys A: Math. Gen. 30 (1997), 5869-5876.

[18] E. T. Whittaker and G. N. Watson, A Course of Modern Analysis, Cambridge University Press, London, 1935.

[19] H. Yoshida, A criterion for the nonexistence of an additional integral in Hamiltonian systems with a homogeneous potential, Phys. D 29 (1987), 128-142.

[20] H. Yoshida, Nonintegrability of the truncated Toda lattice Hamiltonian at any order, Comm. Math. Phys. 116 ( 1988), 529-538.

[21] H. Yoshida, A. Ramani and B. Grammaticos, Nonintegrability of the fourth-order truncated Toda Hamiltonian, Phys. D 30 (1988), 151-163.

[22] H. Żołądek, The Monodromy Group, Monografie Matematyczne 67, Birkhäuser, Basel, 2006. 